Check for updates

Cite this: J. Mater. Chem. C, 2022, 10,688

Received 14th October 2021, Accepted 24th November 2021

DOI: $10.1039 / \mathrm{d} 1 \mathrm{tc0} 4930 \mathrm{e}$

rsc.li/materials-c

\section{A scintillating nanoplatform with upconversion function for the synergy of radiation and photodynamic therapies for deep tumors $\dagger$}

\author{
Yansong Feng, (D) ${ }^{\mathrm{ab}}$ Xiaomeng Liu, ${ }^{\mathrm{a}}$ Qiqing Li, ${ }^{\mathrm{b}}$ Shilin Mei, ${ }^{\mathrm{a}}$ Kefan Wu, ${ }^{\mathrm{b}}$ Jun Yuan, ${ }^{\mathrm{b}}$ \\ Langping Tu, ${ }^{c}$ Ivo Que, ${ }^{d}$ Filippo Tamburini, ${ }^{e}$ Fabio Baldazzi, ${ }^{e}$ Alan Chan, \\ Luis J. Cruz, ${ }^{d}$ Jing Zuo, ${ }^{\text {bf }}$ Changjiang Yao (iD ${ }^{a}$ and Hong Zhang (D) $\star^{b}$
}

\begin{abstract}
Collaborative therapy is regarded as an effective approach in increasing the therapeutic efficacy of cancer. In this work, we have proposed and validated the concept of upconversion lumienscence image guided synergy of photodynamic therapy (PDT) and radiotherapy (RT) for deep cancer, via a specially designed nanoplatform integrating near infrared (NIR) light activated luminescence upconversion and $\mathrm{X}$-ray induced scintillation. Upon NIR light irradiation, the nanoplatform emits highly monochromatic red light solely for imaging the targeted cancer cells without triggering therapy; however, when the irradiation turns to a low dose of X-rays, scintillation will occur which induces effectively the PDT destroying the cancer cells together with X-ray induced RT. The novel theranostic nanoplatform is constructed in such a way that the interactions between the upconversion core and the outmost scintillating shell are blocked effectively by an inert layer between them. This structural design not only enables a nearly perfect excitation energy delivery $(\sim 100 \%$ at a spectral overlapping wavelength of $\sim 540 \mathrm{~nm}$ ) from the outermost scintellating layer to the surface-anchored photosensitizers and so a maximum yield of radical oxygen species, but also achieves a strong NIR induced upconversion luminescence for imaging. Since PDT and RT attack different parts of a cancer cell, this synergy is more effective in destroying cancer than a single therapy, resulting in the reduction of the $\mathrm{X}$-ray irradiation dosage. As a proof of principle, the theranostic effect is validated by in vitro and in vivo experiments, exhibiting the great potential of this sort of nanoplatform in deep cancer treatment.
\end{abstract}

\section{Introduction}

Photodynamic therapy (PDT) is a clinical treatment of certain types of cancer and other diseases. ${ }^{1,2}$ Compared with the traditional radiotherapy (RT, including endogenous and exogenous ones), ${ }^{3}$ PDT has the advantages of being noninvasive, causing less damage/pain, etc. ${ }^{4-6}$ and involves the

\footnotetext{
${ }^{a}$ State Key Laboratory of Explosion Science and Technology, School of Mechatronical Engineering, Beijing Institute of Technology, 100081, Beijing, China

${ }^{b}$ Van't Hoff Institute for Molecular Sciences, University of Amsterdam, Science Park 904, 1098 XH Amsterdam, The Netherlands.E-mail: h.zhang@uva.nl ${ }^{c}$ State Key Laboratory of Luminescence and Applications, Changchun Institute of Optics, Fine Mechanics and Physics, Chinese Academy of Sciences, 130033, Changchun, China

${ }^{d}$ Translational Nanobiomaterials and Imaging, Department of Radiology, Leiden University Medical Center, 2333 ZA, Leiden, The Netherlands

${ }^{e}$ Percuros B.V., Zernikedreef 8, 2333 CL Leiden, The Netherlands

${ }^{f}$ Key Laboratory of Automobile Materials (Ministry of Education), College of Materials Science and Engineering, Jilin University, 130025, Changchun, China. E-mail: jzuo@jlu.edu.cn

$\dagger$ Electronic supplementary information (ESI) available. See DOI: 10.1039/d1tc04930e
}

administration of photosensitizers (PSs) followed by photoactivation; the treatment efficacy depends heavily on the lesion site because of the penetration limit of the excitation light which are primarily ultraviolet (UV) or visible (Vis) light. ${ }^{7-9}$ The clinical application of PDT has been restricted mainly to superficial lesions (e.g. skin cancer). ${ }^{10-13}$ Besides optical fiber, progress on the penetration issue of PDT has mainly been focused on upconversion, thanks to the advancement of the chemistry of nanomaterials. These materials contribute to abundant impressive bio-imaging applications because they were able to convert photons from the near infrared (NIR) to shorter wavelength regions, which greatly increases the penetration depth in tissues. ${ }^{14-18}$ Although there have been reports on upconversion imaging of cancerous tissue located more than one centimeter deep, PDT of the deep cancer remains a tough challenge because therapy usually requires a much higher irradiation dosage than imaging. ${ }^{19-21}$

Recently, a new modality has emerged which uses X-rays instead of UV/Vis as the energy source to initiate PDT (i.e. X-PDT). ${ }^{22-24} \mathrm{X}$-PDT is suitable to treat deep cancer due to 
its "unlimited" penetration depth in tissue. ${ }^{25-27}$ More interestingly, utilizing X-rays as the energy source, it is possible to combine the therapeutic effects of PDT and RT (radiotherapy), achieving a "one plus one greater than two" output, since these two therapies deal with different parts of malignant tumor cells. $^{28,29}$ In PDT: radical oxygen species (ROS), typically singlet oxygen, are generated that cause photocytotoxic damage of the cell membranes, whereas in RT: ionizing radiation induces cell death via nuclear DNA damage. ${ }^{30-33}$ However, this attractive synergy strategy is not yet defined as a clinical modality due to certain limitations. Firstly, the structure and composition of the nanomaterials must have the ability to convert effectively $\mathrm{X}$-ray energy into UV/Vis segments which can respond to photosensitive agents. ${ }^{34-37}$ Secondly, accurately defining the NP targeted cancer locations is the prerequisite for controlling the duration and dosage of the X-ray exposure at a safe level. ${ }^{29,38,39}$ Embedding a real-time optical imaging function on the carrier is thus necessary.

To deal with these issues, employing lanthanide (Ln) fluoride nanocrystals as the host of carriers is a hopeful strategy. Benefitting from the recent progress of the synthetic protocol, the morphology, components, and structure of this type of nanomaterial are able to be well tailored, ${ }^{40,41}$ which provides abundant opportunities for the accurate optimization of the cure effect through a man-made structure design. Moreover, this nanocrystal is one of the very limited choices as a suitable host for both RT\&X-PDT synergy therapy and upconversion luminescence (UCL) imaging, where the switching of the functions can be easily achieved via tuning the excitation wavelength as well as the doped Ln elements. ${ }^{42,43}$ More specifically, (1) under the excitation of X-rays, effective synergy treatment of RT and X-PDT will be achieved through constructing a nonradiative luminescence resonant energy transfer (LRET) between specific $\mathrm{Ln}\left(\right.$ e.g., $\left.\mathrm{Tb}^{3+}, \mathrm{Gd}^{3+}\right)$ and a surface anchored photosensitizer (e.g. Rose Bengal, RB), and (2) before the treatment, employing NIR excitation will bring in real-time and innoxious UCL imaging (at the UV/Vis region) from the different Ln dopants in NPs (the combinations of $\mathrm{Nd}^{3+} / \mathrm{Yb}^{3+}$ and $\left.\mathrm{Er}^{3+} / \mathrm{Tm}^{3+} / \mathrm{Ho}^{3+}\right) .{ }^{44-46}$ which provides a reliable strategy for image-guided therapy in deep cancer.

In this work, we have developed a new UCL-image-guided synergistic RT\&X-PDT nanoplatform (UIRPPs) based on lanthanide ion doped fluorides $\mathrm{NaErF}_{4}: 0.5 \% \mathrm{Tm} @ \mathrm{NaYF}_{4} @ \mathrm{NaGdF}_{4}$ : $15 \% \mathrm{~Tb}$ (Scheme 1). This structure is superior in several aspects: (i) for the first time, a core-shell-shell design is introduced to combine the two desired functions (i.e., UCL imaging and RT\&X-PDT therapy) together. Furthermore, the necessity of a sufficiently thick $\mathrm{NaYF}_{4}$ medium layer $(\sim 2.5 \mathrm{~nm})$ is determined, which blocks the harmful interference between the two functional areas (i.e., the internal core and the outermost layer, respectively). (ii) The spherical shell shaped distribution of the activator of scintillation $(\mathrm{Tb})$ ensures a shorter working distance in X-PDT treatment, which also increases the efficiency of LRET up to nearly $100 \%$. (iii) A recently reported improved synthesis strategy by our group is adopted here. ${ }^{41}$ The results indicated that by minimizing the negative effect of $\mathrm{OH}^{-}$

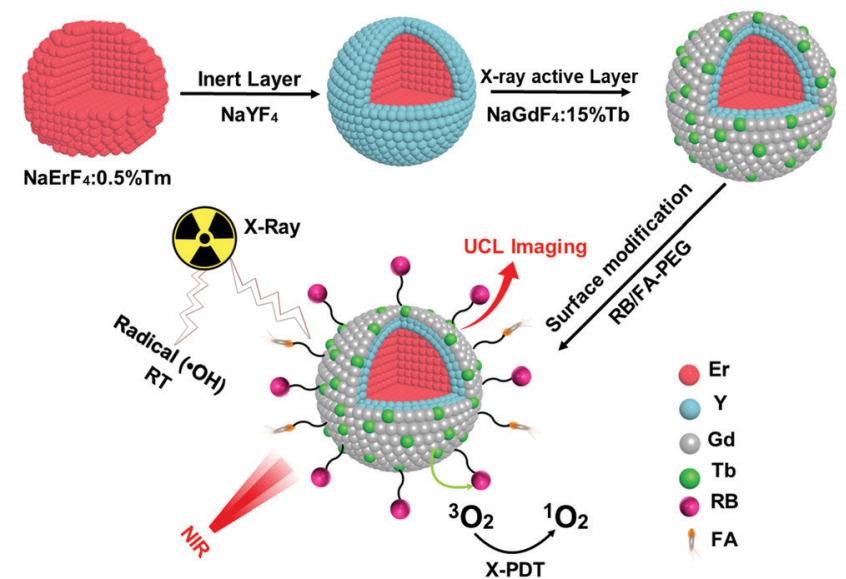

Scheme 1 Schematics of the synthesis and structure of UIRPPs and the principle of UCL imaging under NIR light irradiation and X-ray activated synergistic RT\&X-PDT monitored with red luminescence imaging.

impurities inside the NPs via a "dry" solvothermal method, the UCL intensity of NPs can be improved significantly. To the best of our knowledge, our success in constructing the highly effective, ultra-small nanoplatforms (diameter of $\sim 17 \mathrm{~nm}$ ) for UCL-image guided synergistic RT\&X-PDT is an enlightening endeavor without precedent.

\section{Results and discussion}

\section{Synthesis and characterization}

The structure and synthesis route of UIRPPs are shown in Scheme 1. The tailored core-shell-shell (CSS) structure is synthesized by a dry solvothermal method. From the transmission electron microscopy (TEM) results the average diameters of the core $\left(\mathrm{NaErF}_{4}: 0.5 \% \mathrm{Tm}\right)$ and the CSS are determined to be $\sim 9 \mathrm{~nm}$ (Fig. 1a) and $\sim 17 \mathrm{~nm}$ (Fig. 1b and c), respectively, from which $\sim 2.5 \mathrm{~nm}$ inert layer $\left(\mathrm{NaYF}_{4}\right)$ and $\sim 1.5 \mathrm{~nm}$ thick outermost layer $\left(\mathrm{NaGdF}_{4}: 15 \% \mathrm{~Tb}\right)$ can be readily obtained.
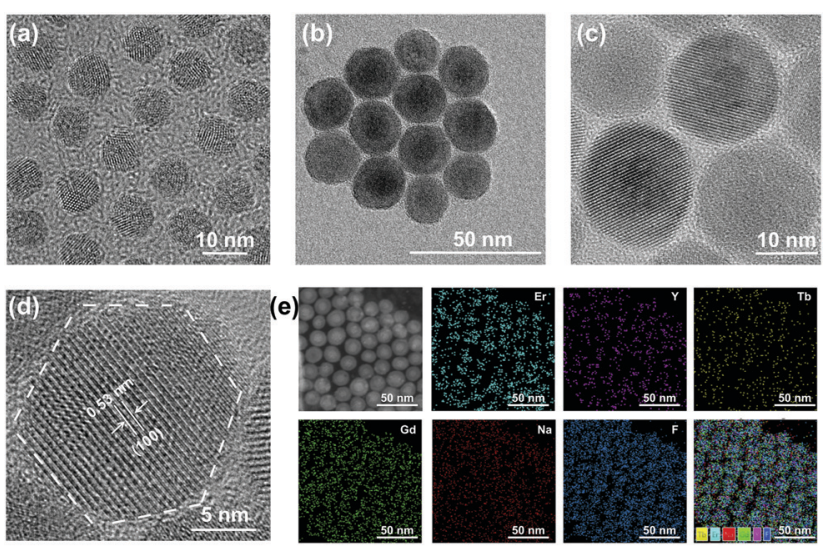

Fig. 1 Characterization of the core and CSS NPs. (a) TEM image of the core $\mathrm{NaErF}_{4}: 0.5 \% \mathrm{Tm}$; (b) TEM images and (c) HRTEM image of the CSS NPs; (d) HRTEM image of an individual CSS NP; (e) HAADF-STEM image of the CSS NPs (left-up) and the STEM-EDS element mapping (Er, Y, Tb, Gd, $\mathrm{Na}$ and F) images of the CSS NPs. 
Furthermore, the high-resolution TEM image (HRTEM) of an individual particle (Fig. 1d) exhibits clear lattice fringes of the hexagonal phase crystal (100) plane with a spacing of $0.53 \mathrm{~nm}$. From X-ray powder diffraction (XRD) (Fig. S1, ESI $\dagger$ ), it can be seen that both the core and the CSS NPs are pure hexagonal phases. The elementary distributions of the CSS nanostructure (Fig. 1e) obtained from the high-angle annular dark field scanning transmission electron microscopy (HAADF-STEM) and scanning transmission electron microscopy with energydispersive X-ray spectroscopy (STEM-EDS) are in line with the $\mathrm{NaErF}_{4}: \mathrm{Tm} @ \mathrm{NaYF}_{4} @ \mathrm{NaGdF}_{4}: \mathrm{Tb} \mathrm{CSS}$ structure.

\section{Optical and nanostructure properties}

The construction of the CSS nanostructure has undergone a three-step optimization. Firstly, different from the most common UCNPs (e.g. sensitizer $\mathrm{Yb} / \mathrm{Nd}$ co-doped with activator $\mathrm{Er} /$ Tm/Ho), which usually exhibit multi-color UCL spectra, in this work, we have chosen the unique $\mathrm{NaErF}_{4}: 0.5 \% \mathrm{Tm}$ component as the imaging core. ${ }^{47}$ As shown in Fig. 2a, this structure is characterized by the monochromatic narrow-band upconversion red emission around $650 \mathrm{~nm}$ with a full width at half maximum (FWHM) of $\sim 11 \mathrm{~nm}$ under the excitation of $800 \mathrm{~nm}$, $980 \mathrm{~nm}$ or $1530 \mathrm{~nm}$. The doped $\mathrm{Tm}^{3+}$ was introduced to quench the green UCL emission (Fig. S2, $\mathrm{ESI} \dagger$ ). In this case, UCL spectra do not overlap with the absorption of the surface anchored photosensitizer (RB, Fig. S3, ESI $\dagger$ ) which guarantees the invalidation of the possible side effect i.e., activating an unwanted PDT effect during the UCL imaging period. Secondly, we focused on the optimization of the inert intermediate layer thickness. Obviously, the intermediate layer (i.e., $\mathrm{NaYF}_{4}$ ) plays a key role not only in minimizing the harmful interactions between $\mathrm{Er}^{3+}$ ions in the core and the surface-related quenching

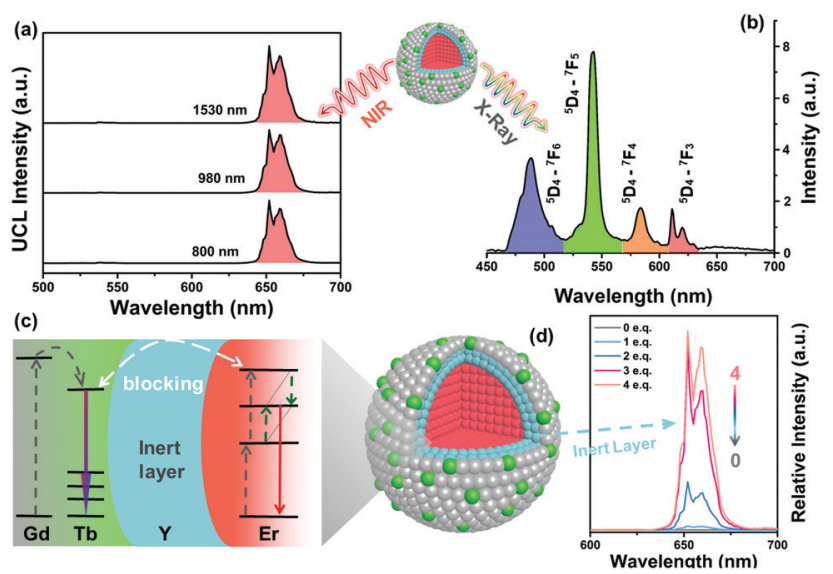

Fig. 2 Optical characterization of NPs with NIR and X-rays. (a) The UCL spectra of NPs under laser excitation at $800 \mathrm{~nm}\left(10 \mathrm{~W} \mathrm{~cm}^{-2}\right), 980 \mathrm{~nm}$ (10 $\mathrm{W} \mathrm{cm}^{-2}$ ) and $1530 \mathrm{~nm}\left(1 \mathrm{~W} \mathrm{~cm}^{-2}\right)$, respectively; (b) the emission spectrum of NPs under X-ray irradiation; (c) schematic diagram of energy transfer interactions subject to NIR or X-ray irradiation; (d) inert layer thickness dependent UCL of NPs under $980 \mathrm{~nm}$ laser excitation. Marked number $(0,1,2,3$, and 4$)$ is the ratio of the inert $\mathrm{NaYF}_{4}$ layer equivalent (eq.) of the core. centers, e.g. surface defects, ligands, solvent molecules etc., but also in blocking the energy transfer pathway between the interior core and the outermost X-ray active layer, thus enhancing the local density of excitation energy (Fig. 2c). As shown in Fig. 2d and Fig. S4 (ESI $\dagger$ ), the core-shell-shell molar ratio in this Er@Y@Gd/Tb structure was varied from 1:0:3 to 1:4:3, and the optimized ratio is determined to be $1: 3: 3$ (layer thickness is $\sim 2.5 \mathrm{~nm}$ ), where the UCL intensities reach saturation with the thinnest layer. Finally, it is also worth noticing that the synthesis of CSS NPs adopts a new dry approach, which was able to exclude the residual $\mathrm{H}_{2} \mathrm{O} / \mathrm{OH}^{-}$defects in NPs efficiently. ${ }^{41}$ As a result, it improves the UCL intensities by approximately 10 times (Fig. S5, ESI $\dagger$ ), which greatly facilitates the high resolution of red imaging and low excitation power density. When the excitation switches to X-rays, the NPs exhibit the characteristic emission of $\mathrm{Tb}^{3+}$ (Fig. 2b) which overlaps reasonably well with RB absorption (Fig. S3, ESI $\dagger$ ). The excited $\mathrm{Tb}^{3+}$ in the outmost layer can thus effectively transfer its energy to the surface anchored RB through nonradiative resonant energy transfer for PDT, partly due to the short energy transfer distance, whereas its red scintillation $\left(\right.$ e.g. $\left.{ }^{5} \mathrm{D}_{4}-{ }^{7} \mathrm{~F}_{3}\right)$ can serve for imaging to in situ monitor the therapy.

To functionalize these NPs further for X-ray mediated PDT applications, the as-synthesized oleic acid (OA) capped NPs were first stirred with $0.1 \mathrm{M} \mathrm{HCl}$ to wash out the surface ligands OA, and then coated with poly(allylamine) (PAAm) to form NPs$\mathrm{NH}_{2}$ via ligand exchange. As shown in Fig. 3a and b, amino group modification increases the particle diameter and the zeta potential from $\sim 26 \mathrm{~nm}$ to $\sim 52 \mathrm{~nm}$ and from $+5.5 \mathrm{mV}$ to $+30 \mathrm{mV}$, respectively, as determined from dynamic light scattering. These changes evidenced that amine groups were successfully anchored on the surface of NPs. Subsequently, photosensitizer RB and cancer targeting moiety folic acid (FA) were covalently conjugated with $\mathrm{NPs}-\mathrm{NH}_{2}$ to form NPs-RB/FA, followed by surface modification with PEG succinimidyl carbonate (PEG-SC) to form UIRPPs to improve further the biocompatibility and specificity. The diameter and zeta potential of the UIRPPs are $\sim 77 \mathrm{~nm}$ and $\sim+17 \mathrm{mV}$, respectively. The successful preparation of UIRPPs is further supported by FTIR spectra (Fig. 3c). The OA-capped NPs (NPs) exhibit the asymmetric and symmetric stretching vibrations of methylene around 2921 and $2854 \mathrm{~cm}^{-1}$, as well as the characteristic of the carboxylic groups around 1556 and $1462 \mathrm{~cm}^{-1}$. PAAm coating exhibits a $\mathrm{N}-\mathrm{H}$ stretching vibration band around $1660 \mathrm{~cm}^{-1}$ whereas the characteristic peaks of OA disappear. Conjugation with a RB molecule brings in new bands around $1662 \mathrm{~cm}^{-1}$ (CO) and $1546 \mathrm{~cm}^{-1}(\mathrm{~N}-\mathrm{H})$ of secondary amide. It deserves noting that the NIR laser would not activate $\mathrm{RB}$ and these modifications have negligible influence on the UCL intensity and its stability, as witnessed by the ten-day trajectory of the UCL spectra of UIRPPs dispersed in water (Fig. S6, ESI $\dagger$ ).

Regarding therapy, this multilayer structure of UIRPPs is key for an effective nonradiative energy transfer from donors inside the nanoparticles to the surface anchored PSs. ${ }^{36,48}$ The X-ray active $\mathrm{Tb}^{3+}$ ions are doped solely in the outermost layer with thickness less than $2 \mathrm{~nm}$, which indicates that $\mathrm{Tb}^{3+}$ ions have a 

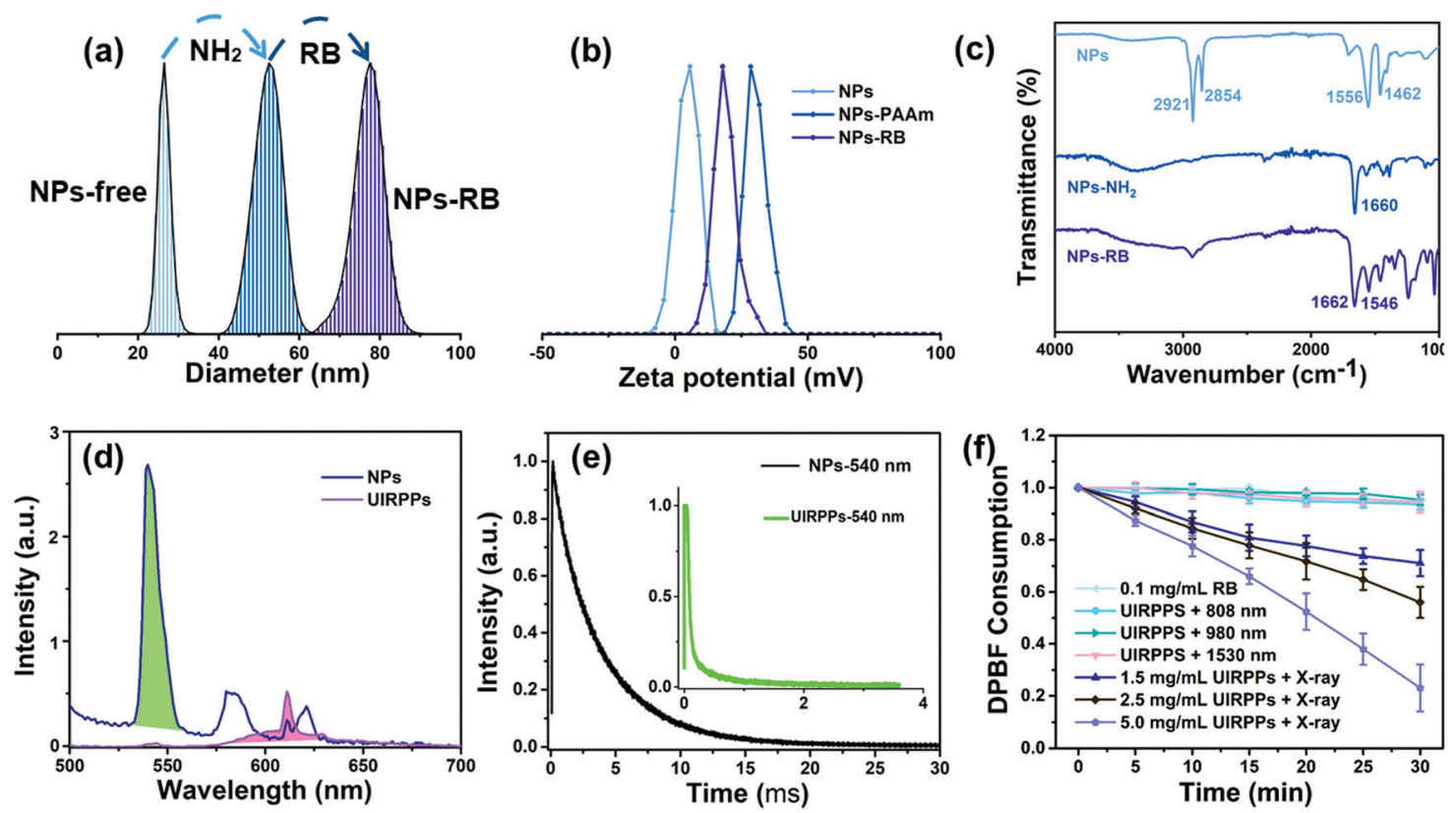

Fig. 3 Stepwise surface modification of UIRPPs and ${ }^{1} \mathrm{O}_{2}$ using NPs as transducers. The diameter (a), zeta potential (b), and FTIR (c) of NPs without ligands (NPs-free), NPs-PAAm, and NPs-RB; (d) the luminescence spectra of NPs and UIRPPs under $254 \mathrm{~nm}$ excitation; (e) the dynamic curves of NP emission (540 nm, black line) and UIRPP emission ( $540 \mathrm{~nm}$, green line) with excitation at $378 \mathrm{~nm}$; (f) relative DPBF consumption of control groups, including the group incubated with $0.1 \mathrm{mg} \mathrm{mL}^{-1} \mathrm{RB}$, the group with $2.0 \mathrm{mg} \mathrm{mL}^{-1}$ UIPRRs irradiated with $800 \mathrm{~nm}$ laser, the group with $2.0 \mathrm{mg} \mathrm{mL}^{-1}$ UIPRRs irradiated with $980 \mathrm{~nm}$ laser, the group with $2.0 \mathrm{mg} \mathrm{mL}^{-1}$ UIPRRs irradiated with $1530 \mathrm{~nm}$ laser, the group with $1.5 \mathrm{mg} \mathrm{mL}^{-1}$ UIRPPs irradiated with X-rays; the group with $2.5 \mathrm{mg} \mathrm{mL}^{-1}$ UIRPPs irradiated with X-rays, and the group with $5.0 \mathrm{mg} \mathrm{mL}^{-1}$ UIRPPs irradiated with X-rays; $n=3$.

spherical shell shape distribution (the 15 mol\% doping concentration of $\mathrm{Tb}^{3+}$ is optimized from the previous reports). ${ }^{36}$ This distribution is conductive to transfer energy from the excited $\mathrm{Tb}^{3+}$ to the PSs because the number of effective energy donors is maximized and the energy transfer distance is the shortest. Based on the CSS structure and high surface-to-volume ratio of NP, PSs loading efficiency is estimated up to $12 \%(\mathrm{w} / \mathrm{w})$ via covalent bonding. From the emission spectra of UIRPPs (Fig. 3d), the disappearance of $\mathrm{Tb}^{3+}$ emission in the blue and green range indicates the occurrence of an effective energy transfer from $\mathrm{Tb}^{3+}$ to $\mathrm{RB}$, which is also supported by the lifetime change of ${ }^{5} \mathrm{D}_{4}-{ }^{7} \mathrm{~F}_{5}$ transition at $540 \mathrm{~nm}$ (Fig. 3e), varied from $3.73 \mathrm{~ms}$ (without $\mathrm{RB}$ ) to $0.04 \mathrm{~ms}$ (with $\mathrm{RB}$ ). The energy transfer efficiency is calculated to be close to $100 \%$.

In addition, one RB molecule has four iodine atoms, which would also contribute to X-ray absorption for radiotherapy. Quantification of ${ }^{1} \mathrm{O}_{2}$ production of UIRPPs irradiated by NIR laser or X-rays was realized with the aid of an oxygen detector 1,3-diphenylisobenzofura (DPBF). As shown in Fig. 3f, Fig. S7 and S8 (ESI $\dagger$ ), the DPBF consumption of $2 \mathrm{mg} \mathrm{mL}^{-1}$ UIRPP aqueous solution remains almost constant with time upon NIR light irradiation $(800 \mathrm{~nm}, 980 \mathrm{~nm}$, or $1530 \mathrm{~nm})$ of $0.7 \mathrm{~W} \mathrm{~cm}{ }^{-2}$, indicating that ${ }^{1} \mathrm{O}_{2}$ generated by NIR laser irradiation in UIRPPs can be ignored. This demonstrated that RB cannot be activated with the UCL core upon NIR laser irradiation as there was no overlap between the monochromatic red UC emission and absorption of RB. On the other side, due to significant overlap between the X-ray spectrum of NPs and the absorption of RB (Fig. S3, ESI $\dagger$ ), it is hypothesized that when nanoparticles are irradiated by X-rays, Tb can transfer energy to $\mathrm{RB}$ for ${ }^{1} \mathrm{O}_{2}$ production. The DPBF absorption, however, does decline once UIRPPs upon X-ray irradiation, even at a low concentration $\left(1.5 \mathrm{mg} \mathrm{mL} \mathrm{m}^{-1}\right) .{ }^{1} \mathrm{O}_{2}$ generation exhibits linear dependence on UIRPP concentration and irradiation time, signifying a constant ${ }^{1} \mathrm{O}_{2}$ generation rate. These results show that to produce ${ }^{1} \mathrm{O}_{2}$, NPs, RB and X-rays all need to be present, corroborating our hypothesis that ${ }^{1} \mathrm{O}_{2}$ production is a result of the LRET system. Collectively, these results indicate unambiguously the very interesting application potential of UIRPPs in minimal phototoxic imaging and highly efficient X-PDT.

\section{UCL imaging and X-PDT in vitro and in vivo}

To assess the application in cancer theranostics, UCL imaging and synergistic RT\&X-PDT of UIRPPs were conducted in vitro. Fig. S9 (ESI $\dagger$ ) shows the viability of a breast cancer cell line (MCF-7) subjected to treatments. There is no obvious cytotoxicity of MCF-7 cells either when incubated with UIRPPs, even at a concentration as high as $400 \mu \mathrm{g} \mathrm{mL}^{-1}$, or upon NIR laser irradiation ( $800 \mathrm{~nm}, 980 \mathrm{~nm}$, or $1530 \mathrm{~nm})$. As expected, 8 hours after the incubation of MCF-7 cells with UIRPPs $\left(100 \mu \mathrm{g} \mathrm{mL} L^{-1}\right)$, the cells emitted bright red UCL signals around the nuclei upon laser irradiation at 980 or $800 \mathrm{~nm}$ (Fig. S10, ESI $\dagger$ ). It is worth noting that there is no green UCL, indicating that we have reached the desired monochromic red UCL for imaging. The NPs were modified with FA to reinforce the cellular uptake efficiency via FA-receptor-mediated endocytosis. The positive targeting was successful after incubation for $8 \mathrm{~h}$ as is shown in Fig. 4a where the UCL signal intensifies with increasing 


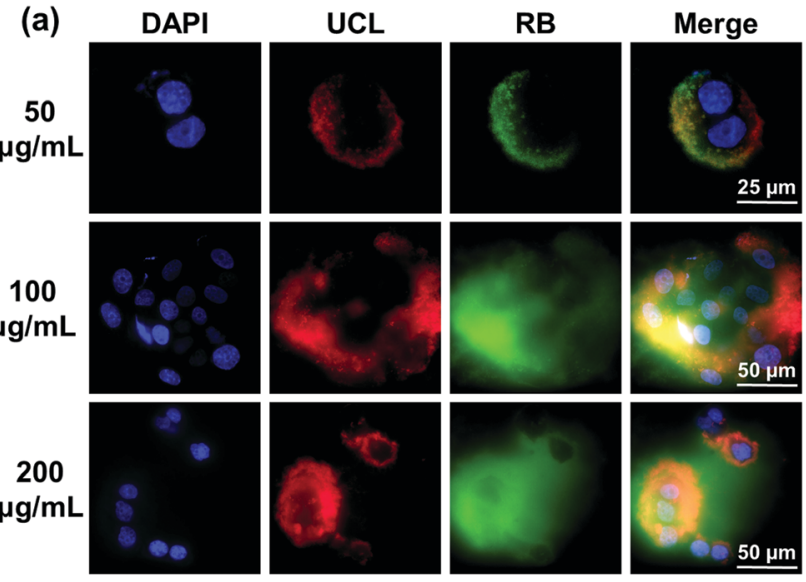

(b)

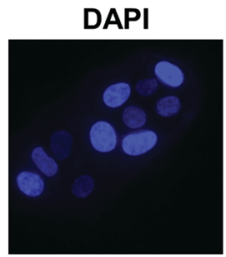

UCL-RB

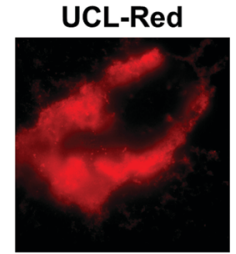

RB
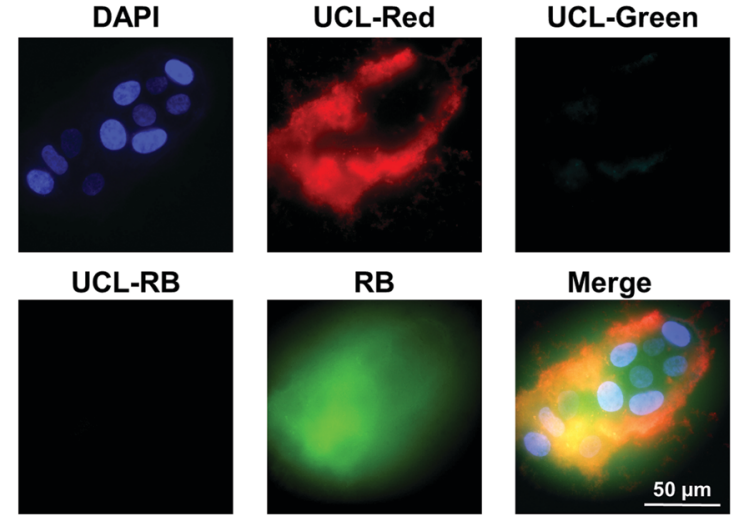

Merge

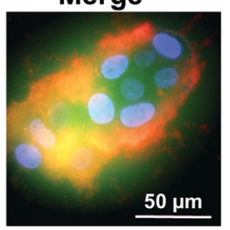

Fig. 4 In vitro imaging results. (a) The confocal images of MCF-7 cells incubated for $8 \mathrm{~h}$ with $50 \mu \mathrm{g} \mathrm{mL}^{-1}, 100 \mu \mathrm{g} \mathrm{mL}^{-1}$ and $200 \mu \mathrm{g} \mathrm{mL}^{-1}$ UIRPPs, respectively, where the nuclei are stained with DAPI. The images with $650 \mathrm{~nm}$ UCL under $980 \mathrm{~nm}$ laser irradiation. RB images with $590 \mathrm{~nm}$ fluorescence under $540 \mathrm{~nm}$ laser irradiation; (b) the different aisle confocal images of MCF-7 cells incubated with UIRPPs under $980 \mathrm{~nm}$ irradiation. UCL-Red, UCL-Green and UCL-RB images at $650 \mathrm{~nm}, 540 \mathrm{~nm}$ and $590 \mathrm{~nm} U \mathrm{CL}$, respectively. RB images with $590 \mathrm{~nm}$ fluorescence under $540 \mathrm{~nm}$ laser irradiation.

concentration of UIRPPs. When the concentration of UIRPPs is between 50 and $200 \mu \mathrm{g} \mathrm{mL}{ }^{-1}$ UCL imaging maintains a high resolution. In order to highlight the advantage of UCL imaging, fluorescence imaging using RB emission under $540 \mathrm{~nm}$ laser irradiation is compared (Fig. 4a). The latter is blurrier mainly due to the scattering of the irradiation light. Fig. S11 (ESI $\dagger$ ) shows the confocal images of Z-scan to exhibit the endocytosis and the high resolution of UCL imaging. Photoactivity of RB subjected to NIR laser irradiation is shown in Fig. 4b where neither UCL green nor RB signal appears, indicating that therapeutic function is not activated during UCL imaging and thus there is no serious photodamage.

MTS assay has been used to evaluate the noninvasive UCL imaging and the synergistic RT\&X-PDT of UIRPPs. As shown in Fig. 5a, irradiation of NIR laser $(800 \mathrm{~nm}, 980 \mathrm{~nm}$, or $1530 \mathrm{~nm})$ does not induce the death of MCF-7 cells statistically, implying the lack of sufficient ${ }^{1} \mathrm{O}_{2}$ generation. In contrast, upon a low dosage of X-ray irradiation ( $80 \mathrm{kV}, 1.5 \mathrm{~Gy}$ ), a significant dropdown of cell viability occurs and is shown in Fig. 5b. Regarding the "UIRPPs + X-ray" group, the number of the apoptotic cells has a positive relationship with the concentration of UIRPPs and the synergistic RT\&X-PDT results in the apoptosis of $\sim 80 \%$ of the cells when incubated with $400 \mu \mathrm{g} \mathrm{mL} \mathrm{m}^{-1}$ UIRPPs. Fig. 5c shows the flow cytometry analysis of the apoptosis of MCF-7 cells experiencing synergistic RT\&X-PDT, where Annexin-VFITC is used to stain apoptotic cells and propidium iodide (PI) is used to stain necrosis cells. The two groups including the MCF-7 cells incubated with saline or UIRPPs plus the $980 \mathrm{~nm}$ laser irradiation and those incubated with UIRPPs plus $980 \mathrm{~nm}$ laser irradiation retained very high survival rates. When irradiated with X-rays, however, the apoptosis of MCF-7 cells obviously occurs. In the "NPs + X-ray" group, about $30 \%$ of the dead cells are exclusively induced by X-rays since there are no PSs in this group. However, in the "UIRPP" groups, the cell survival rate is only $38.3 \%, 26.5 \%$, or $15.6 \%$ cells after irradiation with X-rays corresponding to a UIRPP concentration of 50 , 100 , or $200 \mu \mathrm{g} \mathrm{mL}^{-1}$, respectively. The cell death ratio of $84.4 \%$ is much higher than that of a single X-ray treatment, demonstrating the promising effect of the inherent synergistic RT\&XPDT. These data illustrate the power of the noninvasive UCL imaging and highly effective synergistic RT\&X-PDT in cancer treatment, as well as the huge potential role that UIRPP may play in it.

Furthermore, the UCL imaging and antitumor efficacy were assessed in vivo. For UCL imaging, the mice were intravenously injected with $100 \mu \mathrm{L}$ of UIRPPs at a concentration of $10 \mathrm{mg} \mathrm{mL} \mathrm{m}^{-1} .0 \mathrm{~h}, 1 \mathrm{~h}$ and $24 \mathrm{~h}$ after injection, the mice were exposed to the nontherapeutic $980 \mathrm{~nm}$ laser, as shown in Fig. 6a. After $1 \mathrm{~h}$ the mice had already exhibited UCL imaging at the tumor sites, and strong UCL imaging was observed in the tumor area after $24 \mathrm{~h}$. For the X-PDT efficacy, the mice were randomly divided into 4 groups $(n=3)$ : "PBS" control group injected with PBS; "UIRPPs" group injected with UIRPPs; "PBS + X-ray" group for the RT only; "UIRPPs + X-ray" group for the RT\&X-PDT. Each mouse was injected with $65 \mu \mathrm{L}$ of PBS or UIRPPs (10 $\mathrm{mg} \mathrm{mL}^{-1}$ ) intravenously, respectively. On the basis of effective cellular inhibition in vitro, the in vivo antitumor efficacy upon X-ray irradiation was able to utilize an ultralow X-ray dose (1 Gy). As shown in Fig. 6a and $b$, the "UIRPPs + X-ray" group has the most significant tumor inhibition after 14 days. In addition, the "PBS + X-ray" group also had reduced tumor growth compared to the PBS control group. In contrast, tumors in the "PBS" and "UIRPPs" groups grow rapidly implying a lack of sufficient inhibition effect. There is no systemic reduction in body weight upon treatment with UIRPPs and/or X-rays (Fig. S12, ESI $\dagger$ ). These results suggest that synergistic RT\&X-PDT based on UIRPPs has an apparent inhibition effect on cancer.

\section{Conclusions}

We have developed an effective ultrasmall theranostic nanoplatform for photo-switchable NIR image-guided synergistic RT\&X-PDT for deep cancer. The multilayer nanoplatform based 


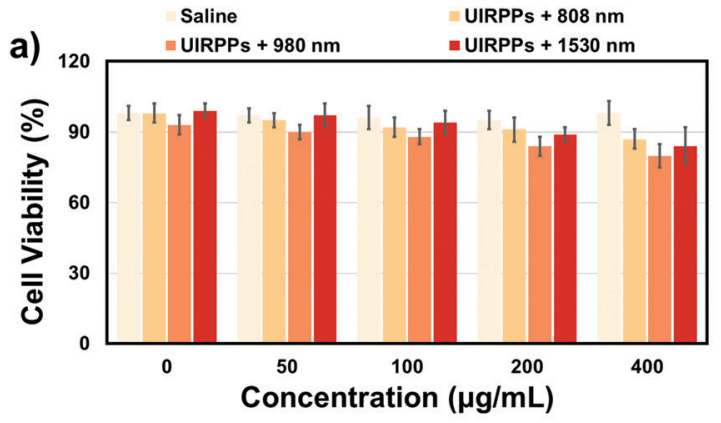

c)
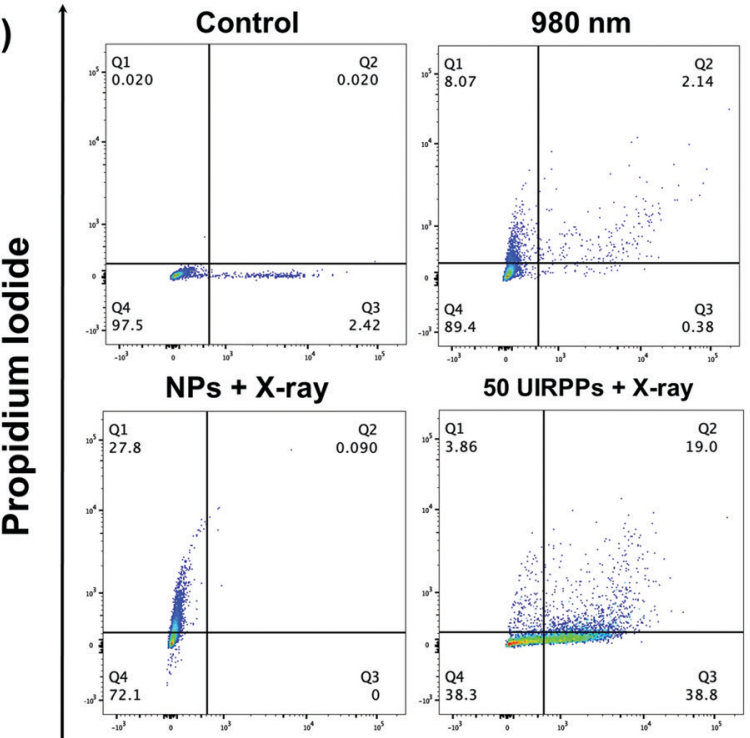
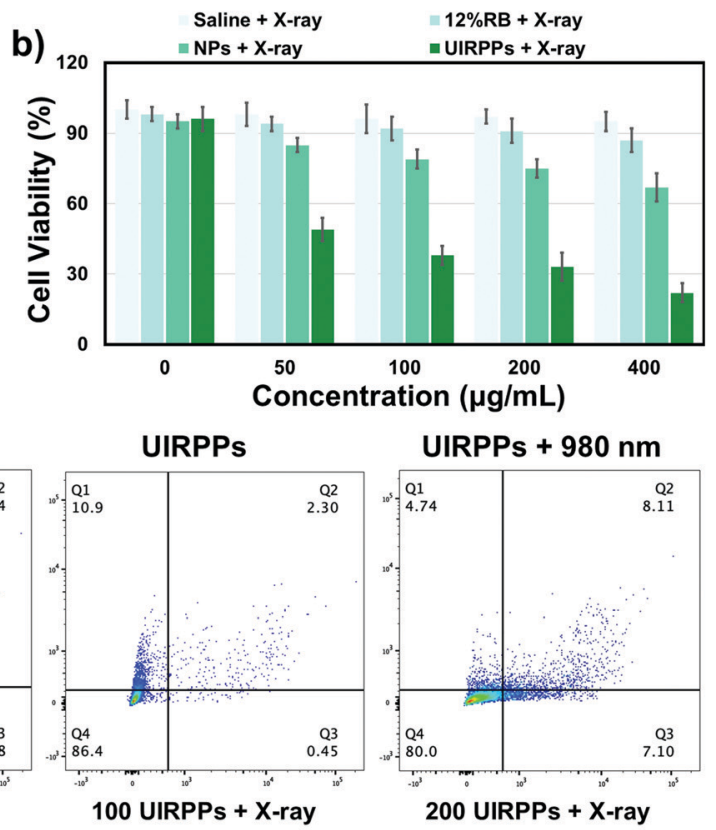

UIRPPs $+980 \mathrm{~nm}$

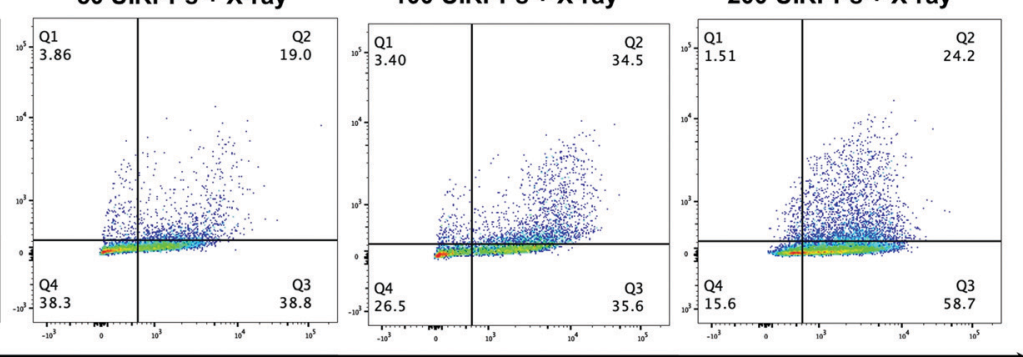

Annexin V-FITC

Fig. 5 Results of in vitro X-PDT. (a) The viability of cells $(n=5)$ incubated with UIRPPs at different concentrations, subjected to irradiation with a NIR laser $(800 \mathrm{~nm}, 980 \mathrm{~nm}$, or $1530 \mathrm{~nm}$, respectively). (b) The cell viability $(n=5)$ of different groups, including the one incubated with saline, with different concentrations of RB, with NPs or UIRPPs plus X-ray irradiation; (c) apoptosis of MCF-7 cell groups, including the one incubated with saline, incubated with UIRPPs, incubated with UIRPPS plus $980 \mathrm{~nm}$ irradiation, under $980 \mathrm{~nm}$ laser irradiation only, incubated with NPs plus X-ray irradiation, and incubated with UIRPPs at different concentrations (50,100 or $200 \mu \mathrm{g} \mathrm{mL}^{-1}$ ) plus X-ray irradiation. The MCF-7 cells are stained with Annexin V-FITC and analyzed by FAC Scan.

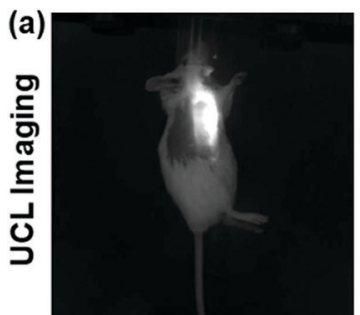

$\mathrm{Oh}$

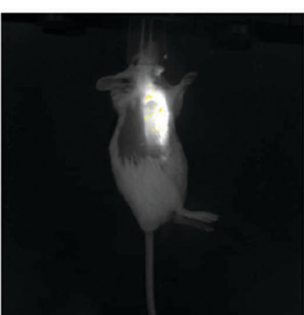

$1 \mathrm{~h}$

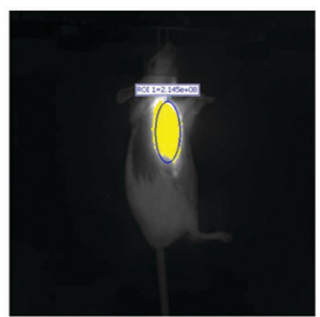

$24 \mathrm{~h}$

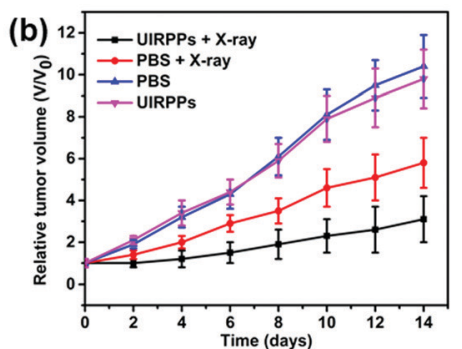

Fig. 6 In vivo imaging and antitumor results of RT\&X-PDT. (a) In vivo UCL imaging of tumor-bearing mouse upon $980 \mathrm{~nm}$ laser irradiation at $0 \mathrm{~h}, 1 \mathrm{~h}$ and $24 \mathrm{~h}$ after intravenous injection. (b) Tumor growth trajectory. Tumor-bearing mice $(n=3)$ were intravenously treated with PBS, UIRPPs, or NP, or PBS, UIRPPs and combined with 1 Gy X-ray irradiation. After the treatments, tumors were weighted every 2 days.

on lanthanide fluoride following a novel dry synthetic approach enables a strong monochromatic deep red UCL under NIR light irradiation for imaging. Under X-ray irradiation, it has prolific ${ }^{1} \mathrm{O}_{2}$ generation when conjugated with proper photosensitizers ascribed to the specially designed structure which enables highly efficient resonant energy transfer. The nanostructure guarantees a clear separation of the imaging and therapeutic functions. Aided with a tumor-targeting agent FA, the UIRPPs demonstrate an effective accumulation in cancer cells and high anticancer ability when subjected to low-dose X-ray irradiation 
due to the synergistic RT and X-PDT. Furthermore, small size and specific targeting agents are conducive to biocompatibility and biosafety. Also, the careful structure design minimizes the potential phototoxicity during imaging/diagnosis. This work sheds light on the precise administration of nanomedicine in the treatment of cancer and other major diseases, with a much safer implementation and better treatment effect of X-rays.

\section{Author contributions}

Y. F.: project design, preparation of nanocomplex, experimental data analysis and discussion, and manuscript writing. X. L., Q. L. and S. M.: sample characterization and data analysis. K. W., J. Y., and L. T.: data analysis and discussion. F. T., F. B. and L. C.: cell experiments. I. Q. and A. C.: mice experiments. C. Y.: data analysis. J. Z.: sample characterization, data analysis and discussion. H. Z.: project design and administration, data analysis and discussion, and manuscript writing.

\section{Conflicts of interest}

There are no conflicts to declare.

\section{Acknowledgements}

This work was financially supported by Netherlands Organization for Scientific Research in the framework of the Fund New Chemical Innovation under grant no. 731.015.206 and TTW perspective project MEDPHOT, EU H2020-MSCA-ITN-ETN Action program, ISPIC, under grant no. 675743, EU H2020MSCA-RISE-2017 Action program, CANCER, under Grant no. 777682, and National Science Fund of China under Grant no. 62105031, Beijing Institute of Technology Research Fund Program for Young Scholars, Fundamental Research Funds for the Central Universities, JLU.

\section{References}

1 S. S. Lucky, K. C. Soo and Y. Zhang, Chem. Rev., 2015, 115, 1990-2042.

2 C.-K. Lim, J. Heo, S. Shin, K. Jeong, Y. H. Seo, W.-D. Jang, C. R. Park, S. Y. Park, S. Kim and I. C. Kwon, Cancer Lett., 2013, 334, 176-187.

3 M. E. Davis, Z. Chen and D. M. Shin, Nat. Rev. Drug Discovery, 2008, 7, 771-782.

4 M. Ethirajan, Y. Chen, P. Joshi and R. K. Pandey, Chem. Soc. Rev., 2011, 40, 340-362.

5 D. E. J. G. J. Dolmans, D. Fukumura and R. K. Jain, Nat. Rev. Cancer, 2003, 3, 380-387.

6 T. J. Dougherty, C. J. Gomer, B. W. Henderson, G. Jori, D. Kessel, M. Korbelik, J. Moan and Q. Peng, J. Natl. Cancer Inst., 1998, 90, 889-905.

7 Y. Jin, H. Wang, X. Li, H. Zhu, D. Sun, X. Sun, H. Liu, Z. Zhang, L. Cao, C. Gao, H. Wang, X.-J. Liang, J. Zhang and X. Yang, ACS Appl. Mater. Interfaces, 2020, 12, 26832-26841.
8 P. Agostinis, K. Berg, K. A. Cengel, T. H. Foster, A. W. Girotti, S. O. Gollnick, S. M. Hahn, M. R. Hamblin, A. Juzeniene, D. Kessel, M. Korbelik, J. Moan, P. Mroz, D. Nowis, J. Piette, B. C. Wilson and J. Golab, Ca-Cancer J. Clin, 2011, 61, 250-281. 9 L. Cheng, C. Wang, L. Feng, K. Yang and Z. Liu, Chem. Rev., 2014, 114, 10869-10939.

10 F. S. De Rosa and M. V. L. B. Bentley, Pharm. Res., 2000, 17, 1447-1455.

11 N. C. Zeitouni, A. R. Oseroff and S. Shieh, Mol. Immunol., 2003, 39, 1133-1136.

12 R. R. Allison, G. H. Downie, R. Cuenca, X.-H. Hu, C. J. H. Childs and C. H. Sibata, Photodiagn. Photodyn. Ther., 2004, 1, 27-42.

13 S. B. Brown, E. A. Brown and I. Walker, Lancet Oncol., 2004, 5, 497-508.

14 D. K. Chatterjee and Z. Yong, Nanomedicine, 2008, 3, 73-82. 15 N. M. Idris, M. K. Gnanasammandhan, J. Zhang, P. C. Ho, R. Mahendran and Y. Zhang, Nat. Med., 2012, 18, 1580-1585.

16 W. Fan, P. Huang and X. Chen, Chem. Soc. Rev., 2016, 45, 6488-6519.

17 G. Tian, Z. Gu, L. Zhou, W. Yin, X. Liu, L. Yan, S. Jin, W. Ren, G. Xing, S. Li and Y. Zhao, Adv. Mater., 2012, 24, 1226-1231.

18 J.-G. Piao, L. Wang, F. Gao, Y.-Z. You, Y. Xiong and L. Yang, ACS Nano, 2014, 8, 10414-10425.

19 J. Tian, L. Ding, H.-J. Xu, Z. Shen, H. Ju, L. Jia, L. Bao and J.-S. Yu, J. Am. Chem. Soc., 2013, 135, 18850-18858.

20 J. Peng, L. Zhao, X. Zhu, Y. Sun, W. Feng, Y. Gao, L. Wang and F. Li, Biomaterials, 2013, 34, 7905-7912.

21 Z. Gu, L. Yan, G. Tian, S. Li, Z. Chai and Y. Zhao, Adv. Mater., 2013, 25, 3758-3779.

22 W. Chen and J. Zhang, J. Nanosci. Nanotechnol., 2006, 6, 1159-1166.

23 J. Takahashi and M. Misawa, Nanobiotechnology, 2007, 3, 116-126.

24 J. P. Scaffidi, M. K. Gregas, B. Lauly, Y. Zhang and T. Vo-Dinh, ACS Nano, 2011, 5, 4679-4687.

25 G. Lan, K. Ni, R. Xu, K. Lu, Z. Lin, C. Chan and W. Lin, Angew. Chem., 2017, 129, 12270-12274.

26 A. Kamkaew, F. Chen, Y. Zhan, R. L. Majewski and W. Cai, ACS Nano, 2016, 10, 3918-3935.

27 K. Lu, C. He, N. Guo, C. Chan, K. Ni, G. Lan, H. Tang, C. Pelizzari, Y.-X. Fu, M. T. Spiotto, R. R. Weichselbaum and W. Lin, Nat. Biomed. Eng., 2018, 2, 600-610.

28 X. Yu, X. Liu, W. Wu, K. Yang, R. Mao, F. Ahmad, X. Chen and W. Li, Angew. Chem., 2019, 131, 2039-2044.

29 L. Larue, A. Ben Mihoub, Z. Youssef, L. Colombeau, S. Acherar, J. C. André, P. Arnoux, F. Baros, M. Vermandel and C. Frochot, Photochem. Photobiol. Sci., 2018, 17, 1612-1650.

30 W. Sun, T. Shi, L. Luo, X. Chen, P. Lv, Y. Lv, Y. Zhuang, J. Zhu, G. Liu, X. Chen and H. Chen, Adv. Mater., 2019, 31, 1808024.

31 G. D. Wang, H. T. Nguyen, H. Chen, P. B. Cox, L. Wang, K. Nagata, Z. Hao, A. Wang, Z. Li and J. Xie, Theranostics, 2016, 6, 2295-2305.

32 X. Zou, M. Yao, L. Ma, M. Hossu, X. Han, P. Juzenas and W. Chen, Nanomedicine, 2014, 9, 2339-2351. 
33 C. Zhang, K. Zhao, W. Bu, D. Ni, Y. Liu, J. Feng and J. Shi, Angew. Chem., Int. Ed., 2015, 54, 1770-1774.

34 W. Chen, J. Biomed. Nanotechnol., 2008, 4, 369-376.

35 H. Chen, G. D. Wang, Y.-J. Chuang, Z. Zhen, X. Chen, P. Biddinger, Z. Hao, F. Liu, B. Shen, Z. Pan and J. Xie, Nano Lett., 2015, 15, 2249-2256.

36 W. Zhang, X. Zhang, Y. Shen, F. Shi, C. Song, T. Liu, P. Gao, B. Lan, M. Liu, S. Wang, L. Fan and H. Lu, Biomaterials, 2018, 184, 31-40.

37 J. Zhao, L. Duan, A. Wang, J. Fei and J. Li, Wiley Interdiscip. Rev.: Nanomed. Nanobiotechnol., 2020, 12, e1583.

38 J. Bernier, E. J. Hall and A. Giaccia, Nat. Rev. Cancer, 2004, 4, 737-747.

39 L. Song, P.-P. Li, W. Yang, X.-H. Lin, H. Liang, X.-F. Chen, G. Liu, J. Li and H.-H. Yang, Adv. Funct. Mater., 2018, 28, 1707496.

40 C. Homann, L. Krukewitt, F. Frenzel, B. Grauel, C. Würth, U. Resch-Genger and M. Haase, Angew. Chem., Int. Ed., 2018, 57, 8765-8769.
41 Y. Feng, Z. Li, Q. Li, J. Yuan, L. Tu, L. Ning and H. Zhang, Light: Sci. Appl., 2021, 10, 105.

42 Y. Feng, H. Chen, Y. Wu, I. Que, F. Tamburini, F. Baldazzi, Y. Chang and H. Zhang, Biomaterials, 2020, 230, 119637.

43 F. Ahmad, X. Wang, Z. Jiang, X. Yu, X. Liu, R. Mao, X. Chen and W. Li, ACS Nano, 2019, 13, 10419-10433.

$44 \mathrm{X}$. Wu, G. Chen, J. Shen, Z. Li, Y. Zhang and G. Han, Bioconjugate Chem., 2015, 26, 166-175.

45 D. Yang, C. Li and J. Lin, Nanomedicine, 2015, 10, 2573-2591.

46 H. Xing, W. Bu, S. Zhang, X. Zheng, M. Li, F. Chen, Q. He, L. Zhou, W. Peng, Y. Hua and J. Shi, Biomaterials, 2012, 33, 1079-1089.

47 Q. Chen, X. Xie, B. Huang, L. Liang, S. Han, Z. Yi, Y. Wang, Y. Li, D. Fan, L. Huang and X. Liu, Angew. Chem., Int. Ed., 2017, 56, 7605-7609.

48 L. Huang, Z. Li, Y. Zhao, J. Yang, Y. Yang, A. I. Pendharkar, Y. Zhang, S. Kelmar, L. Chen, W. Wu, J. Zhao and G. Han, Adv. Mater., 2017, 29, 1604789. 